(2)

\title{
Role of adalimumab in the management of children and adolescents with juvenile idiopathic arthritis and other rheumatic conditions
}

REVIEW

\section{Katherine Anne B Marzan \\ Division of Rheumatology, Children's Hospital Los Angeles, Los Angeles, CA, USA}

This article was published in the following Dove Press journal:

Adolescent Health, Medicine and Therapeutics

19 June 2012

Number of times this article has been viewed

\begin{abstract}
Treatment of children and adolescents with juvenile idiopathic arthritis and other pediatric rheumatic diseases has evolved. Where once there was only a limited arsenal of medications, with significant side effects and inadequate efficacy, today, with an increased understanding of the pathogenesis of these diseases, there is a wider variety of more targeted and effective treatments. TNF- $\alpha$ is a cytokine involved in a number of inflammatory pathways in pediatric rheumatic diseases. The emergence of biologic modifiers that target TNF- $\alpha$ has been pivotal in providing the ability to deliver early and aggressive treatment. Adalimumab, a recombinant monoclonal antibody to TNF- $\alpha$, is an important therapeutic option, which affords children and adolescents with chronic illnesses an improved quality of life.

Keywords: adalimumab, juvenile idiopathic arthritis, TNF- $\alpha$, pediatric, rheumatic diseases, treatment
\end{abstract}

\section{Introduction}

Chronic childhood illnesses, such as juvenile idiopathic arthritis (JIA) and other rheumatic conditions, are a cause of significant morbidity in children and adolescents. ${ }^{1}$ Such illnesses take a toll, not only on the physical well-being of the individual, but also on their emotional and psychosocial health, as well as their growth, development, and peer interactions.

Past treatments for JIA and other rheumatic diseases have included nonsteroidal anti-inflammatory drugs and non-specific immunosuppressive medications, including disease modifying anti-rheumatic drugs, cytotoxic agents, and corticosteroids. ${ }^{2}$ These medications have often been ineffective, or utilized at the cost of significant side effects.

In the last two decades, a greater understanding of the immune system and the mediators of inflammation and tissue damage, coupled with advances in biotechnology, has led to the development of biologic response modifiers for pediatric rheumatic diseases. Biologic targeted therapies are therapeutic agents with biologic properties, including monoclonal antibodies, soluble cytokine receptors, and natural antagonists. ${ }^{2}$ Biologics are genetically engineered proteins and molecules that target specific components of the immune cascade. They inhibit cytokine behavior, cellular activation, and inflammatory gene transcription as antibodies, soluble receptors or natural antagonists. In addition, there are small molecules that specifically inhibit intracellular, cell-cell and cell-matrix interactions, fundamental to the inflammatory and immune process. ${ }^{2,3}$ The majority of biologics bind to soluble

Correspondence: Katherine Anne Marzan Children's Hospital Los Angeles, 4650 Sunset Blvd MS\#60,

Los Angeles, CA, 90027, USA

Tel + I $32336 \mid 2119$

Fax +I 3233618030

Email kmarzan@chla.usc.edu 
cytokines or impede their binding to specific receptors. ${ }^{4,5}$ A significant target in JIA and other rheumatic conditions is the proinflammatory cytokine tumor necrosis factor alpha $(\mathrm{TNF}-\alpha){ }^{4}$

\section{Tumor necrosis factor- $\alpha$ (TNF- $\alpha /$ TNF)}

TNF- $\alpha$ exerts its effects through two distinct receptors found on most cells: TNFR1, a 55-kDa (p55) type 1 receptor, and TNFR2 a $75 \mathrm{kDa}(\mathrm{p} 75)$ type 2 receptor. Activation of TNFR1 primarily leads to inflammation, tissue damage, and cytotoxicity. Activation of TNFR2 primarily leads to T-cell proliferation, T-cell apoptosis, and immunosuppression. When the cells are stimulated, the TNF receptors can be cleaved from the cell surface by proteolysis, and circulate in the cell microenvironment as soluble receptors sTNFR1 and sTNFR2. These soluble receptors endogenously act as inhibitors when they bind to TNF- $\alpha .{ }^{6,7}$ Cellular responses induced by TNF- $\alpha$ may be influenced by the number of cellassociated receptors and the concentrations of sTNFR1 and sTNFR2 receptors in the extracellular fluid. Additionally, the ratio between TNF- $\alpha$ and its soluble receptors in the biologic fluid affects treatment response. ${ }^{8}$

TNF- $\alpha$ is pivotal in the pathogenesis of many inflammatory diseases. Elevated serum levels can be seen in a number of conditions, including JIA, noninfectious inflammatory eye disease, Behçet's Disease, and systemic lupus erythematosus (SLE). TNF- $\alpha$ is produced by many cell types, including macrophages, monocytes, lymphocytes, keratinocytes, and fibroblasts, and can cause damage in various ways. ${ }^{9}$

\section{Adalimumab}

The most experience in the use of TNF inhibitors in pediatric rheumatic diseases has been with JIA. In 1999, etanercept, a genetically engineered soluble TNF receptor, was the first TNF inhibitor approved for JIA. Infliximab, though not formally approved in JIA, is a chimeric monoclonal TNF antibody that is frequently used but requires administration as an infusion. Adalimumab was approved for use in JIA in 2008. ${ }^{10,11}$ Adalimumab has also been used extensively in inflammatory bowel disease (IBD). However such use is beyond the scope of this review.

Adalimumab is a fully-human immunoglobin G1 $\left(\mathrm{IgG}_{1}\right)$ monoclonal antibody specific for human TNF- $\alpha$. Adalimumab is genetically engineered by recombinant DNA phage display technology in a mammalian expression system, resulting in an antibody with human-derived heavy and light chain variable regions, and human $\mathrm{IgG}_{1}-\mathrm{kappa}$ constant regions. It has a molecular weight of approximately $148 \mathrm{kDa}$, and consists of 1330 amino acids. ${ }^{10,12-14}$

Adalimumab binds circulating and cell surface TNF- $\alpha$ and blocks interaction with both TNFR1 and TNFR2 receptors. In vitro, in the presence of complement, adalimumab also lyzes surface membrane TNF-expressing cells. Unlike etanercept, adalimumab does not bind lymphotoxin (TNF- $\beta$ ), and thus provides more specific targeted therapy. ${ }^{12}$

Pharmacokinetic data for healthy children is not available. However, in healthy adults, the maximum serum concentration after a $40 \mathrm{mg}$ adalimumab subcutaneous injection was $4.7 \pm 1.6 \mu \mathrm{g} / \mathrm{mL}$. Maximum concentration was reached at $131 \pm 5$ hours, with a mean terminal half-life of 14 days. ${ }^{12-14}$ In patients 4-17 years of age with JIA, weighing less than $30 \mathrm{Kg}$, the mean steady-state trough serum adalimumab concentrations were $6.8 \mu \mathrm{g} / \mathrm{mL}$ and $10.9 \mu \mathrm{g} / \mathrm{mL}$, respectively, for individuals receiving $20 \mathrm{mg}$ adalimumab subcutaneously every other week as monotherapy, or concomitant with methotrexate. For JIA patients weighing $30 \mathrm{Kg}$ or more, receiving $40 \mathrm{mg}$ adalimumab subcutaneously every other week as monotherapy, or concomitant with methotrexate, the mean steady-state trough serum adalimumab concentrations were $6.6 \mu \mathrm{g} / \mathrm{mL}$ and $8.1 \mu \mathrm{g} / \mathrm{mL}$, respectively. ${ }^{14}$

\section{Juvenile idiopathic arthritis and adalimumab}

JIA is a significant cause of chronic illness in childhood and adolescence, and is the most common pediatric rheumatic disease. Worldwide, an estimated 1.7 to 8.4 million children have arthritis, mostly undiagnosed and untreated. ${ }^{1}$ There is an estimated annual incidence of $0.8-22$ per 100,000 children, and a prevalence of $7-200$ per 100,000, ${ }^{1,10,15-20}$ though one report from Australia gives a prevalence as high as 400 per $100,000 .{ }^{21}$ The broad range is likely to be a reflection of ethnic and geographical variability, as well as the earlier use of different classification systems. ${ }^{1}$

The revised International League of Associations for Rheumatology (ILAR) criteria now comprise the accepted classification of JIA. The ILAR criteria recognize more clinically homogenous groups and include seven types: oligoarthritis, rheumatoid factor (RF) positive polyarthritis, RF negative polyarthritis, systemic arthritis, psoriatic arthritis, enthesitis-related arthritis, and undifferentiated arthritis. ${ }^{1,22}$

JIA is a chronic immunoinflammatory disease that results in synovial hypertrophy and proliferation, and can lead ultimately to bone erosions and destruction. Its long-term consequences of chronic pain, growth retardation, localized growth disturbances, irreversible joint damage, and impaired 
development can be quite devastating. The psychosocial impact on an adolescent can be significant and may impair the individual's ability to find employment. The availability of biologics now makes remission of disease attainable, and improves the long-term outcome for patients. ${ }^{10,11}$

Outcome measures for JIA are based upon the six pediatric core set of criteria (Table 1). ${ }^{15,23}$ The American College of Rheumatology Pediatric 30 pediatric response criteria (ACR Pedi 30) were developed to assess disease activity in JIA. An ACR Pedi 30 response is satisfied by a minimum of $30 \%$ improvement from baseline in a minimum of three out of six components of the core set, with no more than one component worsening by more than $30 \%$. In the same manner, an ACR Pedi 50, 70, and 90 response is defined as 50\%, 70\%, and $90 \%$ improvement, respectively, in a minimum of three of the core set components, with no more than one component worsening by more than $30 \% .^{24}$

The pathogenesis of JIA is still not fully known. It is an autoimmune disease with a complex genetic trait that is postulated to have some B cell dysfunction, though it is primarily thought to involve autoreactive $\mathrm{T}$ cells, with an imbalance in cytokines promoting a proinflammatory response. $^{10}$

TNF- $\alpha$ plays a fundamental role in the pathogenesis of arthritis. When released by synovial macrophages, it stimulates release of interleukin-1 and other cytokines that interact with other cells to stimulate bone and cartilage resorption. It also participates in the promotion of osteoclast differentiation and mature osteoclast activation, leading to bone resorption and bony erosions. TNF- $\alpha$ induces the production of reactive oxygen intermediates, nitric oxide, and prostaglandins, which promote joint inflammation. It also facilitates migration of white blood cells to the synovial tissue, through upregulation of cell adhesion molecules on endothelial cells. In addition, TNF- $\alpha$ is responsible for the release of matrix metalloproteinases that degrade collagen and proteoglycans, which can cause joint space narrowing and cartilage destruction. . $^{1,6,25-28}$

The seminal study on the use of adalimumab in JIA was a multicenter, randomized, stratified, double-blind, placebo-

\section{Table I Pediatric core set criteria ${ }^{23}$}

Physicians global assessment of overall disease activity $(10 \mathrm{~cm}$ visual analog scale)

Parent/patient assessment of overall well-being ( $10 \mathrm{~cm}$ visual analog scale) Functional ability (Childhood Health Assessment Questionnaire)

Number of joints with active arthritis

Number of joints with limited range of motion

Erythrocyte sedimentation rate controlled trial of children and adolescents aged 4-17 years with active moderate polyarticular course JIA. ${ }^{29}$ In the 16-week open-label lead-in period, all patients received $24 \mathrm{mg} / \mathrm{m}^{2}$ adalimumab (maximum $40 \mathrm{mg}$ ) subcutaneously every other week, and were stratified according to methotrexate use. Responders at the end of this period, defined as patients who achieved an ACR Pedi 30, were then randomized, within their stratum, to receive either adalimumab or placebo in the subsequent 32-week double-blind treatment period. Patients who participated in the double-blind treatment period had the option to participate in a 2-year, openlabel adalimumab treatment extension period.

The 16-week, open-label lead-in period was completed by 160 of the 171 JIA patients who enrolled. An ACR Pedi 30 was achieved by $74.4 \%$ on adalimumab monotherapy, and $94.1 \%$ on combination adalimumab and methotrexate treatment. An ACR Pedi 70 was achieved by $59 \%$ and $82 \%$ of patients, respectively. More notably, an almost equal number of patients in both strata achieved an ACR Pedi 90 (28.2\% methotrexate versus $25.6 \%$ non-methotrexate), indicating close-to-clinical remission. Clinical response was rapid, with an ACR Pedi 30 achieved within 2-4 weeks of treatment by $67 \%$ and $77 \%$ of patients in both groups, respectively.

The primary efficacy end point of the 36-week doubleblind portion, demonstrating the percentage of disease flare in the non-methotrexate stratum, was met. A significantly lower percentage of adalimumab-treated patients (43.3\%) than placebo-treated patients $(71.4 \%)$ developed disease flare $(P=0.031)$. The difference was evident, though less pronounced, in the methotrexate-treated group, where $36.6 \%$ and $64.9 \%$, respectively, had disease flare $(P=0.015)$. At the end of 48 weeks, ACR Pedi 30, 50, 70, and 90 responses were achieved by more of the adalimumab- than placebotreated patients, regardless of their methotrexate use. These responses were maintained in the extension phase.

Adalimumab was well tolerated, with only 14 patients having serious adverse events (SAEs), possibly related to its use. The most common adverse events (AEs) were minor infections and injection site reactions.

The use of adalimumab in patients with JIA refractory to prior anti-TNF therapy was evaluated in a prospective, uncontrolled study of six children with JIA, followed over 12-36 months. ${ }^{30}$ Six patients (five systemic JIA, one RF positive polyarthritis), with a polyarticular course and persistent active arthritis despite methotrexate and etanercept and/or infliximab, were given adalimumab $24 \mathrm{mg} / \mathrm{m}^{2}$ subcutaneously every 2 weeks. Although three of the patients discontinued adalimumab due to lack of efficacy, 
improvement (ACR Pedi 30) was achieved by $50 \%$ of the patients. Inclusion in this study was skewed towards patients with refractory disease, and suggests that adalimumab may be of benefit despite prior anti-TNF therapy failure. This study had a number of limitations, which included a small sample size, and a population of patients who were primarily systemic JIA - a subtype that is less responsive to anti-TNF agents. No AEs were reported.

A larger prospective, observational study of 26 Greek patients with refractory JIA (oligoarthritis or polyarthritis), treated with adalimumab and followed over a period of up to 5 years, was done in an intention-to-treat analysis. ${ }^{31} \mathrm{In}$ the first year of treatment, $88 \%$ of patients demonstrated at least an ACR Pedi 30, with 69\% achieving an ACR Pedi 70. As the study progressed, there was a significant drop-off in patients, and only four patients completed the entire 5 years of observation. Thirteen patients (50\%) completed 3 years of the study, with $90 \%$ of these patient still at an ACR Pedi 50 or greater. While the numbers were small, there were two patients who had radiologic improvement after the fourth year of treatment, and two patients who remained stable.

Discontinuation of the study was due to inefficacy in seven patients, and SAEs in two patients. The most serious SAE was a death in an 18 year old, attributed to sepsis following a febrile illness and recent travel abroad. The other SAE was a staphylococcal skin abscess at the site of injection, which responded to surgical drainage and antibiotics. The majority of patients tolerated adalimumab well, with seven patients reporting AEs that were primarily respiratory infections.

Further long-term safety and efficacy trials are currently being conducted. ${ }^{32}$

\section{Ocular inflammatory conditions and adalimumab}

Non-infectious inflammatory uveitis is most commonly idiopathic but can be associated with a number of rheumatologic conditions, notably JIA. Uveitis is characterized by inflammation of all or part of the uveal tract that includes the iris, ciliary body and choroid..$^{33}$ Complications of chronic uveitis include cataracts, glaucoma, synechiae, band keratopathy, and cystoid macular edema. It is the third most common cause of preventable blindness in the developed world with children at an increased risk because of the higher frequency of posterior uveitis compared to adults. ${ }^{34}$

TNF- $\alpha$ is an important amplifying factor in the inflammatory reaction of noninfectious uveitis, and is found in high concentration in the aqueous humor. It has been found to induce polymorphonuclear granulocyte infiltration of the uveal tissue in mice and rats. ${ }^{35}$ Dick et al ${ }^{36}$ noted that inhibition of TNF- $\alpha$ ameliorated intraocular inflammation in experimental autoimmune uveitis. ${ }^{37}$ Adalimumab has been used to treat pediatric uveitis, but there are no randomized, controlled studies.

In a prospective, open-label study Vazquez-Cobian et a ${ }^{37}$ demonstrated decreased ocular inflammation in 13 of 14 children 4-19 years of age with uveitis (five idiopathic, nine JIA-associated), who were treated with weekly subcutaneous adalimumab. Topical corticosteroid use was decreased in $78.5 \%$ of patients; $93 \%$ were able to discontinue, or be weaned from the concomitant immunosuppressive medications. The only AE reported was pain at the injection site. ${ }^{37}$

Biester et $\mathrm{al}^{38}$ did a retrospective analysis of 18 children/ young adults with juvenile uveitis (one idiopathic, 17 JIAassociated), treated with adalimumab $20-40 \mathrm{mg}$ every 2 weeks. The primary outcome parameter was the recurrence rate, with a relapse defined as an increase in cells in the anterior chamber of $2+$ or more. Adalimumab was found to be effective in 16 patients, mildly effective in one patient, and ineffective in one patient. The AEs reported were primarily injection site reactions. One patient developed elevated transaminase levels, and another developed HSV keratitis. ${ }^{38}$

In another retrospective study, the efficacy of adalimumab was evaluated in 20 JIA patients with uveitis, 19 of whom had failed prior anti-TNF therapy. Patients had a mean age of 13.4 years, and mean duration of uveitis of 8.7 years. Improved activity was noted in $35 \%$ of patients, primarily in those who were younger and had a shorter duration of disease. SAEs or complications were not noted. ${ }^{39}$

The role of adalimumab in the prevention of flare recurrences in childhood-refractory chronic uveitis was investigated in an open-label, prospective, comparative, multicenter cohort study. ${ }^{40}$ Thirty-three patients with refractory, vision-threatening, non-infectious active uveitis received either infliximab infusions at $5 \mathrm{mg} / \mathrm{Kg}$, or subcutaneous adalimumab injections at $24 \mathrm{mg} / \mathrm{m}^{2}$ every 2 weeks. The primary outcome was an assessment of time to relapse, after remission had been achieved. The secondary outcomes were: to compare time to remission, time to steroid discontinuation, and number of uveitis relapses, after initiation of anti-TNF treatment. There was no significant difference between the two cohorts (17 infliximab and 16 adalimumab), in terms of time to remission and time to steroid discontinuation. However, the adalimumab group was less likely to flare. At the 40 -month follow-up time point, $60 \%$ of the adalimumab group were in remission, compared to only $19 \%$ of the infliximab group. 
While further long-term and randomized controlled trials still need to be done, adalimumab has shown efficacy in patients with refractory uveitis, and in maintaining remission.

The success of anti-TNF agents in ocular inflammatory diseases such as uveitis ${ }^{38-40}$ has led to its use in orbital myositis. ${ }^{41}$ Orbital myositis is an inflammatory condition that affects the orbital muscles, presenting with pain, orbital swelling, diplopia, nausea, and erythema. Infection, malignancy, and systemic illness must be considered. However, the etiology is often nonspecific and unknown. It is responsive to corticosteroid treatment, but relapses frequently recur, and there is the need for additional immunosuppression. Adams et $\mathrm{al}^{41}$ reported the use of adalimumab in two patients (17 and 13 years old) who had steroid-dependent, recurrent, nonspecific orbital myositis that was refractory to standard immunosuppression. Adalimumab use resulted in clinical improvement, and the ability to wean steroids.

\section{Systemic lupus erythematosus and adalimumab}

Systemic lupus erythematosus (SLE) is an autoimmune disease characterized by dysregulation of the innate and adaptive immune system that can affect multiple organ systems. While more commonly seen in adults, $15 \%-20 \%$ of patients develop SLE before 18 years of age. The mean age of diagnosis of pediatric SLE is $12-13$ years. Disease in childhood is more severe than in adults, with worse longterm outcomes for childhood-onset disease. However, earlier diagnosis and advances in the treatment of pediatric SLE, and its complications, have improved 5-year survival from $82 \%$ to more than $95 \% .{ }^{42,43}$

The role of TNF in SLE disease appears to include both immunoregulatory and proinflammatory effects. The New Zealand Black/White lupus-prone mouse has an inherited relative TNF deficiency, which accelerates autoimmune disease. Treatment of these mice with high-dose recombinant TNF delayed the onset of lupus-like disease. However, experimental lupus mouse models that are not inherently deficient in TNF were noted to have significant increase in TNF of the serum and tissues of mice that had increasing lupus disease activity. Attempts to block TNF in these mouse models reduced inflammatory organ disease. Serum TNF and serum concentrations of soluble TNFR receptors are markedly increased in patients with active SLE. ${ }^{44,45}$ In addition, Malide et al demonstrated TNF- $\alpha$ in the mesangial cells of patients with lupus nephritis. ${ }^{46}$
TNF inhibitor therapy in SLE remains controversial. Formation of SLE-associated autoantibodies has been reported with TNF therapies. There remain concerns, though there have been no reported cases of SLE or lupus-like syndromes in the adalimumab trials for JIA. ${ }^{29}$ There appear to be some clinical benefits to anti-TNF therapy. A pilot study on the use of infliximab showed improvement in some parameters of SLE, ${ }^{47}$ and an open-label study of six patients demonstrated improved proteinuria and arthritis. ${ }^{48}$ There are no reports on the use of adalimumab in pediatric SLE.

\section{Other rare pediatric rheumatic diseases and adalimumab}

Adalimumab has been utilized in a number of other rare autoimmune conditions that also appear to be influenced by TNF- $\alpha$.

Patients with active Behçet's Disease, a relapsing systemic vasculitis, have elevated levels of TNF, soluble TNF receptors, and TNF-producing cells. ${ }^{49-51}$ Behçet's Disease is characterized by recurrent aphthous stomatitis, genital ulceration, uveitis, and thrombophlebitis. It is most common in Japan, Turkey, and the Middle East, but can be seen world wide. Onset in childhood can occur in $5 \%-13 \%$ of patients. ${ }^{52}$ Neurologic involvement may include meningoencephalitis, benign intracranial hypertension, or organic psychiatric disturbances ${ }^{53}$ Studies on uveitis have included a few patients with Behçet's Disease, whose inflammatory eye disease responded to adalimumab. ${ }^{40}$ While there is more experience on the use of infliximab and etanercept, ${ }^{49}$ there have been reports on adalimumab-responsive pediatric Behçet's Disease patients; one patient with neuro-Behçet's Disease showed clinical and MRI improvement. ${ }^{54,55}$

There have been scattered reports on the use of adalimumab in adults with relapsing polychondritis, anti-neutrophil cytoplasmic antibody-associated systemic vasculitis, and sarcoidosis. ${ }^{56-58}$ However, the use of adalimumab in these conditions has not been reported in children.

\section{Safety}

The use of anti-TNF agents has led to better quality of life and clinical outcomes for children and adolescents. However, reports of SAEs in adults make safety a continuing concern. SAEs include tuberculosis (TB) and other opportunistic infections, demyelinating disorders, and SLE and lupuslike syndromes. There have been no reports of these SAEs in children treated with adalimumab. The increased risk of SAEs for adults may be due to risk factors such as smoking, alcohol consumption, and comorbid conditions, such as 
hypertension, diabetes, renal disease, emphysema, and cardiac conditions. ${ }^{59-61}$

The safety of adalimumab has been evaluated through the analysis of large databases. While the data from these studies are primarily from adults, there is some pediatric safety data included. Schiff et $\mathrm{al}^{62}$ assessed the safety of adalimumab in patients with RA, and included data from 10,050 patients, representing 12,506 patient-years of adalimumab exposure. Burmester et $\mathrm{al}^{63}$ went further, by comparing safety data from global trials for multiple indications, which included 171 patients in the JIA trial, with 99 patient-years of adalimumab use.

\section{Infections}

TNF is an important component of the immune system. While it can have a number of deleterious effects, it is also integral to the host's defense, raising the concern for infections, particularly opportunistic ones, with the use of anti-TNF agents. Defense against Mycobacterium tuberculosis, in particular, relies on TNF to contain latent disease and mediate granuloma homeostasis. Not surprisingly, many of the serious infections with adalimumab occurred in patients on concomitant immunosuppressive medications. ${ }^{62}$

Reports of opportunistic infections, apart from TB, in the adult adalimumab clinical trial database included four cases of histoplasmosis and one case each of aspergillosis, nocardia, and atypical mycobacterium. The rate for serious infections in the JIA adalimumab database was 4.04 per 100 patient-years, and did not include any opportunistic infections. Nevertheless, close monitoring for opportunistic infections is imperative, particularly in endemic areas. ${ }^{62-64}$

The most common infections associated with adalimumab use were minor upper respiratory infections. ${ }^{29}$ However, more serious infections have been reported, including herpes simplex virus (HSV) infection $(n=1)$, varicella zoster infection $(n=1)$, pneumonia $(n=1),{ }^{29} \operatorname{HSV}$ keratitis $(n=1),{ }^{38}$ injection site abscess, which improved with drainage and antibiotics $(n=1)$, and sepsis, leading to death $(n=1)$, in a patient who had been on multiple immunosuppressive agents and had traveled abroad. ${ }^{31}$

The TB rate in the earlier adult adalimumab trials, without routine screening for TB, was 1.3 per 100 patient-years. The TB rate under the new protocol, which requires TB screening, isoniazid treatment for patients with a positive skin test, and exclusion of patients with active TB, was 0.33 per 100 patient-years. ${ }^{62}$ No cases of TB were reported in any of the JIA or uveitis studies. ${ }^{29-31,37-40}$ There was one case report of disseminated tuberculosis in a 17 year old with SAPHO syndrome (synovitis, acne, pustulosis, hyperostosis, osteitis), treated with adalimumab. ${ }^{65}$

Evaluation for active and latent TB is imperative prior to treatment with adalimumab. Close follow-up of children on anti-TNF- $\alpha$ therapy, to monitor for tuberculosis, remains important. However, a positive tuberculin skin test does not preclude the use of anti-TNF agents, particularly if they are necessary to prevent disease progression. Completing at least the first month of a 9-month course of isoniazid therapy would be prudent, prior to initiating treatment with adalimumab. ${ }^{66,67}$

\section{Demyelinating disorders}

The use of TNF antagonists in multiple sclerosis proved not only ineffective, but resulted in some reports of increased lesions, and shorter time to flares, which improved with withdrawal of the medication. As such, the development of demyelinating disorders has been a concern. In the adalimumab safety database, there were ten cases in 12,506 patient-years, which included six multiple sclerosis, two Guillan-Barre syndrome, and two nonspecific demyelination. No demyelinating disorders have been reported in the pediatric adalimumab trial. ${ }^{62}$

\section{Malignancies}

The concern for malignancy is always raised with immunosuppressant use. ${ }^{68}$ There have been no reports of malignancies in the pediatric adalimumab clinical trial. ${ }^{29}$ However, in the last decade, the FDA received 48 reports of malignancies in children and adolescents on anti-TNF therapy, who were not in the clinical trials. Twenty-six of these patients had IBD, which is known to carry a higher risk for malignancy. Twenty of these patients had pediatric rheumatic disease, primarily JIA. Only two were on adalimumab (one JIA, one IBD). ${ }^{69}$

Studies have indicated an increased risk of lymphoma in patients with RA. ${ }^{70,71}$ However, the updated report by Wolfe and Michaud did not show evidence of increased lymphoma incidence in patients treated with 10,815 patient-years of anti-TNF therapy. ${ }^{68}$ Nonlymphomatous cancers have not been associated with adalimumab use. ${ }^{64}$ The incidences of lymphoma in the adult adalimumab trials were comparable to those of adalimumab-naive RA patients. ${ }^{62,64}$

The data for underlying risk of malignancy in JIA is less clear. Evaluation for the underlying risk of cancer in patients with JIA, compared to the general population, was done by Simard et al, utilizing the Swedish national database. ${ }^{72}$ Overall, biologic-naive patients with JIA, in the last 40 years, did not have an increased risk of cancer. Interestingly, when 
the data were divided into two 20-year time periods, there was an increased risk of lymphoproliferative cancer in biologicnaive patients over the last 20 years that was not noted in patients identified over the last 40 to 20 years. Another study utilized a US database of two cohorts: biologic-naive JIA patients and non-JIA patients. ${ }^{73}$ The incidence rate of cancer in the JIA cohort (67/100,000 patient-years) was more than twice that of the non-JIA cohort (23.2/100,000 patient-years). In contrast, Bernatsky et al, in their Canadian study, did not find an increased cancer risk in the initial years following a diagnosis of JIA. ${ }^{74}$ A more recent study, by Beukelman et al, evaluated data from 7812 JIA patients, with a total follow-up time of 12,614 patient-years. Malignancy rates for JIA were compared to two cohorts of children without JIA (asthma, and attention deficit hyperactivity disorder). Risk of malignancy in the JIA cohort was also evaluated, based on their medication exposure (methotrexate or leflunamide, TNF inhibitors, and other immunomodulatory agents). Similar to the study by Simard et al, the JIA cohort had an increased risk of malignancy, compared to the non-JIA group. More notably, the treatment of JIA with TNF inhibitors did not appear to be significantly associated with the development of malignancy. ${ }^{75}$

Long-term registries ${ }^{32}$ are in progress that will help clarify this risk, though the use of concomitant immunosuppressive medications will always be a confounding factor. The need to weigh the risks and benefits remains important, taking into account the known risks of poor outcome with inadequate therapy, for JIA and other pediatric rheumatic diseases.

While the greatest concern for adverse events is focused on infections and malignancies, there were other rare AEs noted. These included leucopenia, elevated liver enzymes, and elevated creatinine phosphokinase levels. There have been no reports of the development of liver failure, SLE, or lupus-like syndromes. However, $16 \%$ of patients in the adalimumab JIA clinical trial had at least one positive test for anti-adalimumab antibody, which did not appear to affect efficacy. Concomitant use of methotrexate is not required. ${ }^{14,29}$

Injection site reactions were the most common AEs associated with adalimumab use, particularly a burning sensation with administration. During the open-label lead in period of the JIA clinical trial, injection site reactions were 5.2/27.3 patient-years and 5.67/29.3 patient-years for methotrexate and nonmethotrexate patients, respectively. Additional complaints included erythema, dysethesia, ecchymoses, urticaria, and pruritus. Reactions occurred in the first few months of therapy, but improved over time. ${ }^{10,29}$

\section{Immunizations}

There are no clear guidelines regarding immunizations in pediatric rheumatic diseases. In general, live vaccines are not recommended in patients on immunosuppressive medications, including anti-TNF agents. ${ }^{14,76}$ While inactive vaccines appear safe, the possibility of inadequate response in an immunocompromised host must always be considered. ${ }^{76}$

\section{Summary}

The outcome of pediatric rheumatic disease has improved over the years. A better understanding of the immune system and mediators of damage in pediatric autoimmune diseases, coupled with advancements in biologic therapies, has led to the ability to provide early and aggressive treatment. The efficacy of anti-TNF agents, such as adalimumab, in juvenile idiopathic arthritis has made significant impact on the resolution of clinical symptoms, disease activity on physical examination, and radiographic progression. Clinical remission of disease is not only possible, but is now a treatment goal. When achieved, the improved outcome for adolescents will translate into an adult life with improvement in pain control, daily functioning, social interaction, emotional health, and general well-being. ${ }^{10}$ The success of adalimumab in JIA has led to its use in other pediatric rheumatic diseases. While there are no clinical studies comparable to its use in JIA, adalimumab, nevertheless, may be a therapeutic option in noninfectious inflammatory eye disease and Behçet's Disease. Adalimumab, a well tolerated and efficacious biologic therapy, is part of the therapeutic arsenal that can improve the long-term outcome and quality of life of children and adolescents with JIA, and potentially those with other pediatric rheumatic disease.

\section{Disclosure}

The author reports no conflicts of interest in relation to this work.

\section{References}

1. Petty RE, Cassidy JT. Chronic Arthritis in Childhood. In: Cassidy JT, Petty RE, Laxer RM, Lindsley CB, editors. Textbook of Pediatric Rheumatology, 6th ed. Philadelphia, PA. Elsevier Saunders; 2011:211-235.

2. Shanahan JC, Moreland LW, Robert HC. Upcoming biologic agents for the treatment of rheumatic diseases. Curr Opin Rheumatol. 2003;15(3) 226-236.

3. Isaacs JD. Antibody engineering to develop new anti-rheumatic therapies. Arthritis Res Ther. 2009;11(3):225.

4. Breda L, Del Torto M, De Sanctis S, Chiarelli F. Biologics in children's autoimmune disorders: efficacy and safety. Eur J Pediatr. 2011;170: 157-167.

5. Haines KA. Juvenile Idiopathic Arthritis: therapies in the 21st century. Bull NYU Hosp Jt Dis. 2007;65(3):205-211. 
6. Arend WP. The mode of action of cytokine inhibitors. J Rheumatol. 2002;29 Suppl 65:16-21.

7. Dayer J-M. Interleukin 1 or tumor necrosis factor- $\alpha$ : which is the real target in rheumatoid arthritis. J Rheumatol. 2002;29 Suppl 65: $10-15$.

8. Rooney M, Varsani H, Martin K, et al. Tumor necrosis factor- $\alpha$ and its soluble receptors in juvenile chronic arthritis. Rheumatology. 2000;39: 432-438.

9. Zhu L-J, Yang X, Yu X-Q. TNF- $\alpha$ therapies in systemic lupus erythematosus. J Biomed Biotechnol. 2010;2010:465898.

10. Marzan KAB, Reiff AO. Adalimumab in juvenile rheumatoid arthritis/ juvenile idiopathic arthritis. Expert Rev Clin Immunol. 2008;4(5): $549-558$.

11. Shenoi S, Wallace CA. Tumor necrosis factor inhibitors in the management of juvenile idiopathic arthritis. An evidence-based review. Pediatr Drugs. 2010;12(6):367-377.

12. Keystone E, Haraoui B. Adalimumab therapy in rheumatoid arthritis. Rheum Dis Clin NAm. 2004;30:349-364.

13. Weisman MH, Moreland LW, Furst DE, et al. efficacy, pharmacokinetic, and safety assessment of Adalimumab, a fully human anti-tumor necrosis factor-alpha monoclonal antibody, in adults with rheumatoid arthritis receiving concomitant methotrexate: a pilot study. Clin Ther. $2003 ; 25: 1700-1721$.

14. Humira ${ }^{\circledR}$ (adalimumab). [Package insert] North Chicago, IL: Abbott Laboratories; 2011.

15. Andersson GB. Juvenile arthritis - who gets it, where and when? A review of current data on incidence and prevalence. Clin Exp Rheumatol. 1999;17:367-374.

16. Mielants H, Veys EM, Maertens M, et al. Prevalence of inflammatory rheumatic diseases in an adolescent urban student population, age 12 to 18, in Belgium. Clin Exp Rheumatol. 1993;11:563-567.

17. Laaksonen AL. A prognostic study of juvenile rheumatoid arthritis: analysis of 544 cases. Acta Paediatr Scand. 1966:1-163.

18. Towner SR, Michet CJ Jr, O'Fallon WM, et al. The epidemiology of juvenile arthritis in Rochester, Minnesota, 1960-1979. Arthritis Rheum. 1983;26:1208-1213.

19. Gewanter HL, Roghmann KJ, Baum J. The prevalence of juvenile arthritis. Arthritis Rheum. 1983;26:599-603.

20. Kunnamo I, Kallio P, Pelkonen P. Incidence of arthritis in urban Finnish children: a prospective study. Arthritis Rheum. 1986;29:1232-1238.

21. Manners PJ, Diepeveen DA. Prevalence of juvenile chronic arthritis in a population of 12-year-old children in urban Australia. Pediatrics. 1996;98:84-90

22. Beresford MW. Juvenile Idiopathic Arthritis. New Insights into classification, measures of outcome, and pharmacotherapy. Pediatr Drugs. 2011;13(3):161-173.

23. Giannini EH, Ruperto N, Ravelli A, et al. Preliminary definition of improvement in juvenile arthritis. Arthritis Rheum. 1997;40: 1202-1209

24. Ringold S, Wallace CA. Measuring clinical response and remission in juvenile idiopathic arthritis. Curr Opin Rheumatol. 2007;19: $471-476$.

25. Moore TL. Immunopathogenesis of juvenile rheumatoid arthritis. Curr Opin Rheumatol. 1999;11:377-383.

26. Wilkinson N, Jackson G, Gardner-Medwin J. Biologic therapies for juvenile arthritis. Arch Dis Child. 2003;88:186-191.

27. Ou LS, See LC, Wu CJ, et al. Association between serum inflammatory cytokines and disease activity in juvenile idiopathic arthritis. Clin Rheumatol. 2002;21:52-56.

28. Kutukculer N, Caglayan S, Aydogdu F. Study of pro-inflammatory (TNF- $\alpha$, IL-1 $\alpha$, IL-6) and T-cell-derived (IL-2, IL-4) cytokines in plasma and synovial fluid of patients with juvenile chronic arthritis: correlations with clinical and laboratory parameters. Clin Rheumatol. 1998; 17:288-292.

29. Lovell DJ, Ruperto N, Goodman S, et al. Adalimumab with or without methotrexate in juvenile rheumatoid arthritis. N Engl J Med. 2008;359: $810-820$.
30. Katsicas MM, Russo RA. Use of adalimumab in patients with juvenile idiopathic arthritis refractory to etanercept and/or infliximab. Clin Rheumatol. 2009;28:985-988.

31. Trachana M, Pratsidou-Gertsi P, Pardalos P, Kozeis N, Badouraki M, Kanakoudi-Tsakalidou F. Safety and efficacy of adalimumab treatment in Greek children with juvenile idiopathic arthritis. Scand J Rheumatol. 2011;40:101-107.

32. Abbott Laboratories. Juvenile idiopathic arthritis (JIA) registry (STRIVE). [ClinicalTrials.gov identifier NCT00783510]. US National Institutes of Health. Available from: http://www.clinicaltrials.gov. Accessed January 17, 2012.

33. Sukumaran S, Marzan K, Shaham B, Reiff A. High dose infliximab in the treatment of refractory uveitis: Does dose matter? ISRN Rheumatology. 2012:ID765380.

34. Reiff A. Ocular complications of childhood rheumatic diseases: Uveitis. Curr Rheumatol Rep. 2006;8(6):459-468.

35. Neri P, Zucchi M, Allegri P, Lettieri M, Mariotti C, Giovannini A. Adalimumab (Humira $\left.{ }^{\circledR}\right)$ : a promising monoclonal anti-tumor necrosis factor alpha in ophthalmology. Int Ophthalmolol. 2011;31:165-173.

36. Dick AD, Duncan L, Hale G, Waldmann H, Isaacs J. Neutralizing TNF alpha activity modulates $\mathrm{T}$ cell phenotypes and function in experimental autoimmune uveoretinitis. J Autoimmune. 1998;11:255-264.

37. Vazquez-Cobian LB, Flynn T, Lehman TJA. Adalimumab therapy for childhood uveitis. J Pediatr. 2006;149:572-577.

38. Biester S, Deuter C, Michels H, et al. Adalimumab in the therapy of uveitis in childhood. Br J Ophthalmol. 2006;91:319-324.

39. Tynjälä, P, Kotaniemi K, Lindahl P, et al. Adalimumab in juvenile idiopathic arthritis-associated chronic anterior uveitis. Rheumatology. 2008;47:339-344.

40. Simonini G, Taddio A, Cattalini M, et al. Prevention of flare recurrence in childhood refractory chronic uveitis: an open-label comparative study of adalimumab versus infliximab. Arthritis Care Res. 2011;63(4):612-618.

41. Adams AA, Kazim M, Lehman TJM. Treatment of orbital myositis with adalimumab (Humira). J Rheumatol. 2005;32:1374-1375.

42. Von Scheven E, Bakkaloglu A. What's new in pediatric SLE. Best Pract Res Clin Rheumatol. 2009;23:699-708.

43. Silverman E, Eddy A. Systemic Lupus Erythematosus. In: Cassidy JT, Petty RE, Laxer RM, Lindsley CB, editors. Textbook of Pediatric Rheumatology, 6th ed. Philadelphia, PA. Elsevier Saunders; 2011: 315-343.

44. Rönnblom L, Elkon KB. Cytokines as therapeutic targets in SLE. Nat Rev Rheumatol. 2010;6:339-347.

45. Aringer M, Smolen JS. Efficacy and safety of TNF-blocker therapy in systemic lupus erythematosus. Expert Opin Drug Saf. 2008;7(4): 411-419.

46. Malide D, Russo P, Bendayan M. Presence of tumor necrosis factor alpha and interleukin-6 in renal mesangial cells of lupus nephritis patients. Hum Pathol. 1995;26:558-564.

47. Uppal SS, Hayat SJ, Raghupathy R. Efficacy and safety of infliximab in active SLE: a pilot study. Lupus. 2009;18:690-697.

48. Aringer M, Graninger WB, Steiner G, Smolen JS. Safety and efficacy of tumor necrosis $\alpha$ blockade in systemic lupus erythematosus. Arthritis Rheum. 2004;50(10):3161-3169.

49. Arida A, Fragiadaki K, Giavri E, Sfikakis PP. Anti-TNF agents for Behçet's Disease: analysis of published data on 369 patients. Semin Arthritis Rheum. 2011;41:61-70.

50. Turan B, Gallati H, Erdi H, Gurler A, Michel BA, Villiger PM. Systemic levels of T cell regulatory cytokines IL-10 and IL-12 in Behçet's Disease; soluble TNFR-75 as a biological marker of disease activity. J Rheumatol. 1997;24(1):128-32.

51. Evereklioglu C, Er H, Türköz Y, Cekmen M. Serum levels of TNFalpha, sIL-2R, IL-6, and IL-8 are increased and associated with elevated lipid peroxidation in patients with Behçet's Disease. Mediators Inflamm. 2002;11:87-93.

52. Karincaoglu Y, Borlu M, Toker S, et al. Demographic and clinical properties of juvenile onset Behçet's Disease: a controlled multicenter study. J Am Acad Dematol. 2008;58:597-584. 
53. Ozen S, Petty RE. Behçet's Disease. In: Cassidy JT, Petty RE, Laxer RM, Lindsley CB, editors. Textbook of Pediatric Rheumatology, 6th ed. Philadelphia, PA. Elsevier Saunders; 2011:552-558.

54. Robinson AB, Gallentin WB, Rabinovich CE. Pediatric Neuro-Behçet's Disease responsive to adalimumab. Neurol. 2010;43:291-293.

55. Eleftheriou D, Melo M, Marks SD, et al. Biologic therapy in primary systemic vasculitis of the young. Rheumatology. 2009;48:978-986.

56. Lahmer T, Knopf A, Treiber M, Heemann U, Thuermel K. Treatment of relapsing polychondritis with the TNF-alpha antagonist adalimumab. Clin Rheumatol. 2010;29:1331-1334.

57. Laurino S, Chaudhry A, Booth A, Conte G, Jayne D. Prospective study of TNF- $\alpha$ blockade with adalimumab in ANCA-associated systemic vasculitis with renal involvement. Nephrol Dial Transplant. 2010;25 3307-3314.

58. Callejas-Rubio JL, Ortego-Centeno N, Lopez-Perez L, Benticuaga MN. Treatment of therapy-resistant sarcoidosis with adalimumab. Clin Rheumatol. 2006;25:596-597.

59. Imperato AK, Smiles S, Abramson SB. Long-term risks associated with biologic response modifiers used in rheumatic diseases. Curr Opin Rheumatol. 2004;16:199-205.

60. Desai S, Furst DE. Problems encountered during anti-tumor necrosis factor therapy. Best Pract Res Clin Rheumatol. 2006;20:757-790.

61. Bongartz T, Sutton AJ, Sweeting MJ, et al. Anti-TNF antibody therapy and the risk of serious infections and malignancies. Systematic review and meta-analysis of rare harmful effects in randomized controlled trials. JAMA. 2006;295:2275-2285.

62. Schiff MH, Burmester GR, Kent JD, et al. Safety analyses of adalimumab (HUMIRA) in global clinical trials and US postmarketing surveillance of patients with rheumatoid arthritis. Ann Rheum Dis. 2006;65:889-894.

63. Burmester GR, Mease PJ, Dijkmans BAC, et al. Adalimumab clinical trial safety in multiple indications and reduction in mortality in rheumatoid arthritis. Arthritis Rheum. 2006;54 Suppl 9:S323.

64. Keystone E, Haraoui B. Adalimumab therapy in rheumatoid arthritis. Rheum Dis Clin North Am. 2004;30:349-364.

65. Hess S, Hospach T, Nossal R, Dannecker G, Magdorf K, Uhlemann F. Life-threatening disseminated tuberculosis as a complication of TNF- $\alpha$ blockade in an adolescent. Eur J Pediatr. 2011;170(10):1337-1342.
66. Kilic O, Kasapcopur O, CamciogluY, Cokugras H, Arisoy N, Akcakaya N Is it safe to use anti-TNF- $\alpha$ agents for tuberculosis in children suffering with chronic rheumatic disease? Rheumatol Int. 2011. [Epub ahead of print.]

67. Ayaz AN, Demirkaya E, Bilginer Y, et al. Preventing tuberculosis in children receiving anti-TNF treatment. Clin Rheumatol. 2010;29(4): 389-392.

68. Wolfe F, Michaud K. The effect of methotrexate and anti-tumor necrosis factor therapy on the risk of lymphoma in rheumatoid arthritis in 19,562 patients during 89,710 person-years of observation. Arthritis Rheum. 2007;56:1433-1439.

69. Diak P, Siegel J, la Grenade L, Choi L, Lemery S, McMahon A. Tumor necrosis factor $\alpha$ blockers and malignancy in children. Forty-eight cases reported to the food and drug administration. Arthritis Rheum. 2010; 62(8):2517-2524.

70. Ekstrom K, Hjalgrim H, Brandt L, et al. Risk of malignant lymphoma in patients with rheumatoid arthritis and their first-degree relatives. Arthritis Rheum. 2003;48:963-970.

71. Franklin L, Lunt M, Bunn D, et al. Incidence of lymphoma in a large primary care derived cohort of cases of inflammatory polyarthritis. Ann Rheum Dis. 2006;65:617-622.

72. Simard JF, Neovius M, Hagelberg S, Askling J. Juvenile idiopathic arthritis and risk of cancer. Arthritis Rheum. 2010;62:3776-3782.

73. Harrison M, Nordstrom B, Gu y, et al. Risk of malignancy in biologicsnaïve juvenile arthritis patients. Ann Rheum Dis. 2010;69 Suppl 3:631.

74. Bernatsky S, Rosenberg AM, Oen KG, et al. Malignancies in juvenile idiopathic arthritis: a preliminary report. J Rheumatol. 2011;38: 760-763.

75. Beukelman T, Haynes K, Curtis JR, et al. Rates of malignancy associated with juvenile idiopathic arthritis and its treatment. Arthritis Rheum. 2012;64:1263-1271.

76. Davies K, Woo P. Immunization in rheumatic diseases of childhood: an audit of the clinical practice of British Paediatric Rheumatology Group members and a review of the evidence. Rheumatology. 2002;41 937-941.
Adolescent Health, Medicine and Therapeutics

\section{Publish your work in this journal}

Adolescent Health, Medicine and Therapeutics is an international, peer-reviewed, open access journal focusing on health, pathology, and treatment issues specific to the adolescent age group. All aspects of health maintenance, preventative measures and disease treatmen interventions are addressed within the journal and practitioners from

\section{Dovepress}

all disciplines are invited to submit their work as well as healthcare researchers and patient support groups.. The manuscript management system is completely online and includes a very quick and fair peerreview system. Visit http://www.dovepress.com/testimonials.php to read real quotes from published authors. 